\title{
ZICOS - Neutrinoless Double Beta Decay experiment using Zr-96 with an organic liquid scintillator -
}

\author{
Yoshiyuki FUKUDA* \\ Department of Physics, Faculty of Education, Miyagi University of Education \\ E-mail: fukudaestaff.miyakyo-u.ac.jp
}

Shigetaka MORIYAMA, Katsuki HIRAIDE

Kamioka Observatory, Institute for Cosmic Ray Research, University of Tokyo

\section{Izumi OGAWA}

Department of Applied Physics, Faculty of Engineering, University of Fukui

\section{Takahiro GUNJI, Ryohei HAYAMI, Satoru TSUKADA}

Department of Pure and Applied Chemistry, Faculty of Science and Technology, Tokyo University of Science

\section{Shunsuke KUROSAWA}

Institute for Material Research, Tohoku University

\begin{abstract}
A liquid scintillator containing a tetrakis (isopropyl acetoacetato) zirconium has been developed for ZICOS experiment. In order to reach the sensitivity $T_{1 / 2}^{0 v} \geq 10^{27}$ years, we have to use tone scale of ${ }^{96} \mathrm{Zr}$ and have to reduce $95 \%$ of ${ }^{208} \mathrm{Tl}$ decay background. Using Monte Carlo simulation, we could demonstrate new method using the topological information of Cherenkov light, and could reduce $93 \%$ of ${ }^{208} \mathrm{Tl}$ background with $78 \%$ efficiency for $0 v \beta \beta$ signal. For the discrimination of Cherenkov light, the precise spectral pulse shape from Cherenkov lights was directly measured by using sub-MeV electrons from ${ }^{90} \mathrm{Sr} /{ }^{90} \mathrm{Y}$ beta source. The observed shape was clearly different from the shape of scintillation light. The pulse rise and fall (decay) time for Cherenkov light were $0.8 \mathrm{~ns}$ and $2.5 \mathrm{~ns}$, respectively. They were actually shorter than those times of scintillation light which were also measured by $1.6 \mathrm{~ns}$ and $6.5 \mathrm{~ns}$, respectively. This clear difference of rise time will be used for the pulse shape discrimination in order to select PMT which receive Cherenkov lights, and the topological information due to Cherenkov light will be used for the reduction of backgrounds from ${ }^{208} \mathrm{Tl}$ decay which should be major backgrounds observed around Q-value (3.35MeV) of ${ }^{96} \mathrm{Zr}$ neutrinoless double beta decay.
\end{abstract}

European Physical Society Conference on High Energy Physics - EPS-HEP2019 -

10-17 July, 2019

Ghent, Belgium

${ }^{*}$ Speaker. 


\section{ZICOS experiment}

ZICOS is one of the future experiment for neutrinoless double beta decay. The target nuclei is ${ }^{96} \mathrm{Zr}$ and the $\mathrm{Q}$-value is $3.35 \mathrm{MeV}$, therefore the radioactive backgrounds such as ${ }^{214} \mathrm{Bi}$ in Uranium series and ${ }^{10} \mathrm{C}$, which is spallation product of energetic cosmic muons, could be removed by their lower energy. The conceptional design of ZICOS detector is shown in left panel of Fig.W. The detector consists of spherical frame mounted by 650 of 20 inch photomultiplier (PMT) and inner balloon filled with a liquid scintillator, therefore it is almost similar structure as KamLAND-Zen detector. As reported by KamLAND-Zen[四, non-negligible events were found around 3 - $4 \mathrm{MeV}$, and those were the decay products from ${ }^{208} \mathrm{Tl}$ which was adhere on the surface of inner balloon. Fortunately the Q-value of ${ }^{136} \mathrm{Xe}$ is $2.479 \mathrm{MeV}$ so that those were not backgrounds due to out of range for signal region.

However those events should be quite serious backgrounds for ZICOS experiment. The ZICOS detector will use liquid scintillator containing a tetrakis (isopropyl acetoacetato) zirconium $\left(\mathrm{Zr}(\mathrm{iPrac})_{4}\right)$ inside of inner balloon, and will use pure water for outside of inner balloon. Therefore almost half of ${ }^{208} \mathrm{Tl}$ events observed in KamLAND-Zen should be reduced by missing the energy. However, another half will be unavoidable backgrounds for $96 \mathrm{Zr} 0 v \beta \beta$ signal. In order to remove those backgrounds to reach the sensitivity $T_{1 / 2}^{0 v} \geq 2 \times 10^{27}$ years, we have been developed the reduction technique using topological information from Cherenkov light [2]].
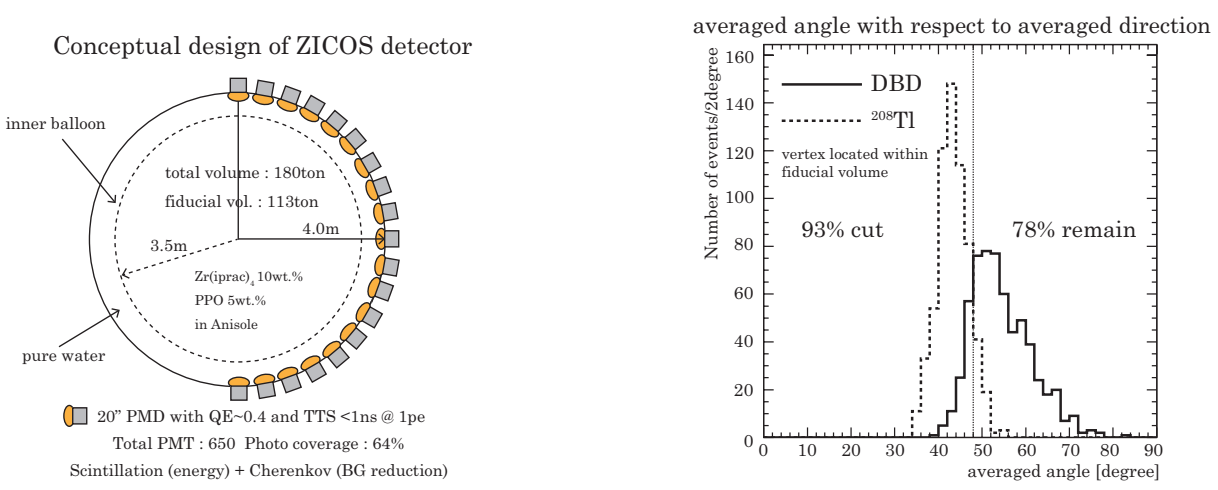

Figure 1:

\section{Discrimination of Cherenkov light using pulse shape}

As described in our previous papers [ [ 3$]$ [䧃], there is a difference of hit pattern of Cherenkov lights between ${ }^{208} \mathrm{Tl}$ backgrounds and $0 \nu \beta \beta$ signals in case of Monte Carlo simulation, and we could reduce about $93 \%$ of ${ }^{208} \mathrm{Tl}$ beta decay events with $78 \%$ efficiency for $0 v \beta \beta$ events using an adequate topological information (defined by averaged angle) from Cherenkov light as shown in right panel of Fig.⿴囗十.

ZICOS detector also should measure the energy as precise as possible in order to reduce background from $2 v \beta \beta$ signals, so that we have to observe scintillation lights for the precise energy 
measurement. Therefore it is necessary to extract the hitted PMT by Cherenkov light among the large yield of scintillation. In this points of view, we have to discriminate PMT whether including Cherenkov light or not using the pulse shape. Generally speaking, Cherenkov radiation is generated by the vibration of an electromagnetic dipole moment, so that the timing spreads during passing time of the charged particle (a few 100 pico seconds). On the other hands, scintillation is the radiation from transition between the exited state and lower state of scintillator atoms, therefore the timing should be decided by nuclear property with a few tenth of nano seconds. This difference of spectral shape at both rise and decay time could be observed. In particular, the difference of rise time should be important because of poor amount of photon from Cherenkov light.

\section{Pulse shape of Cherenkov light and Scintillation using sub-MeV electrons}

In order to observe the rise time of pulse shape precisely, we introduced two equipments. One is photomultiplier which should have a fast timing response for both rise time and transit time spread (TTS). We used both fast PMT Hamamatsu H2431-50 (R2083) and CAEN V1721 digitizer for this measurement.In this paper, we used 1 GS/s ADC for Zero Length Encording (DPP-ZLEplus). These two fast timing spectral measurement would separate the shape of rise time between Cherenkov light and scintillation clearly.

We used $1 \mathrm{MBq}^{90} \mathrm{Sr}$ (half life 28.79 year) for sub-MeV electrons source. The end point energy of electrons from ${ }^{90} \mathrm{Sr}$ is $0.546 \mathrm{MeV}$ so that all electrons could not emit Cherenkov light because of under Cherenkov threshold. On the other hand, ${ }^{90} \mathrm{Y}$ (half life 64 hour) beta decay should be a radioactive equilibrium, then same radiation yield could be expected. The end point energy of electrons from ${ }^{90} \mathrm{Y}$ is $2.280 \mathrm{MeV}$, so that about half of electrons could emit Cherenkov lights. Using electrons above $0.679 \mathrm{MeV}$, we measured the pulse shape for Cherenkov light.
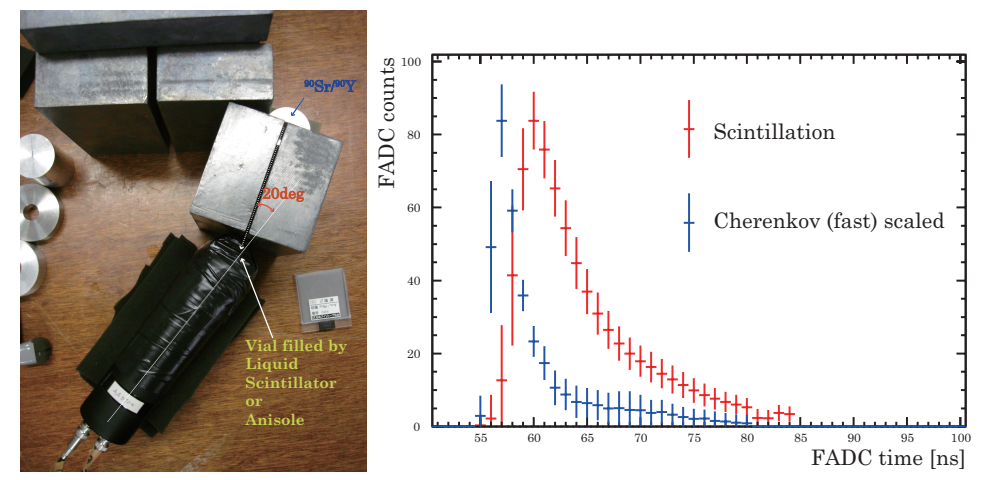

Figure 2:

The left photograph of Fig.[ shows the setup for this measurement. The incident electrons were colimated by Lead block and the direction was fixed by 20 degree with respect to the direction of PMT, because it is easy to induce Cherenkov photon to the PMT. For the measurement of scintillation, this setup was same even though Cherenkov photon was induced to PMT, because of large amount of light yield of scintillation. 


\section{Averaged Pulse shape of Cherenkov light and Scintillation}

In order to obtain the spectral shape, we have to collect signal events and have to make a distribution of FADC counts for each timing bin. Of course, same distribution for backgrounds in case of no source should be subtracted in each timing bin.

At first, we measured the pulse shape for scintillation.Because of high statistics, we could select events with almost same pulse height. Next we measured the pulse shape from Cherenkov light. In this time, the vial which was filled with only Anisole was covered by SC-37 filter in order to observe Cherenkov photons only above $400 \mathrm{~nm}$. For real experiment, Cherenkov photons below $400 \mathrm{~nm}$ should be absorbed by the secondary scintillator such as PPO, therefore only photons above $400 \mathrm{~nm}$ should be observed. Due to environmental backgrounds, we have selected events with almost same pulse height for signal and background. Again FADC count distributions for each timing in case of Cherenkov light were obtained by subtracting the distribution from no source. Using mean and RMS for each distribution, we made averaged pulse shape of Cherenkov light.

The right panel of Fig. $\square$ shows the averaged pulse shape for scintillation and Cherenkov light, respectively. In this figure, the shape of Cherenkov light was scaled to same peak height as scintillation. The observed Cherenkov pulse shape was clearly different from the shape of scintillation. The pulse rise and fall (decay) time for Cherenkov light were $0.8 \mathrm{~ns}$ and $2.5 \mathrm{~ns}$, respectively. They were actually shorter than those times of scintillation light which were also measured by $1.6 \mathrm{~ns}$ and $6.5 \mathrm{~ns}$, respectively. This clear difference of rise time will be used for the pulse shape discrimination in order to select PMT which receive Cherenkov lights, and the topological information such as averaged angle which was described in our previous paper [B] will be used for the reduction of backgrounds from ${ }^{208} \mathrm{Tl}$ decay which should be major backgrounds observed around Q-value (3.35 $\mathrm{MeV}$ ) of ${ }^{96} \mathrm{Zr}$ neutrinoless double beta decay.

\section{Conclusion}

Using sub-MeV electron from ${ }^{90} \mathrm{Sr} /{ }^{90} \mathrm{Y}$ beta source, we successfully measured the actual pulse shapes of Cherenkov light. The measured pulse rise and decay time for Cherenkov light were actually shorter than those times of scintillation. We will develop the pulse shape discrimination in order to distinguish PMT whether receive Cherenkov light or not within 2019 fiscal year. Also we are going to measure an actual energy resolution using special light guide which has almost 60 $\%$ photocoverage, and will measure the topological information using hemisphere detector HUNIZICOS to directly obtain an averaged angle for fixed directional electron.

\section{References}

[1] A.Gando et al. (KamLAND-Zen collaboration), Physical Review Letters 117 (2016) 082503.

[2] Y.Fukuda, Journal of Physics: Conference Series 718 (2016) 062019.

[3] Y.Fukuda, Y.Kamei, Narengerile, A.Obata, S.Moriyama, I.Ogawa, T.Gunji, R.Hayami, S.Tsukada, Bulletin of Miyagi University of Education 52 (2017) 139-147.

[4] Y.Fukuda, Y.Kamei, Narengerile, A.Obata, S.Moriyama, K.HIraide, I.Ogawa, T.Gunji, R.Hayami, S.Tsukada, S.Kurosawa Bulletin of Miyagi University of Education 53 (2018) 183-192. 